\title{
GENERACIÓN AUTOMÁTICA DE RESPUESTAS EN ANÁLISIS MORFOLÓGICO
}

\author{
O. SANTANA - J. PÉREZ - L. LOSADA \\ (Universidad de Las Palmas de Gran Canaria)
}

\begin{abstract}
This paper is concemed with the problem of obtaining a procedure for the automatic generation of texts, inside a defined domain. In the case of study the domain is composed of sentences which result from a Spanish morphological analysis. One important difficulty in order to develop the automatic generation of texts system is to prepare the input data. In the present work the domain is a group of simple results of morphological analysis in natural language. Input data redundancy has been climinated from such group, the essential information has been extracted and the logical structure has been obtained. The possibility to say the same in different ways forces to select the lexicon, to observe the context and to reinforce the control by means of selection criteria with the aim of assuring that the obtained text is correct and formal, objective and concise. The main contribution of this work consists in converting the logical structure into a sentence which represents the information in a cohesive, coherent, comprehensible way and with certain style.
\end{abstract}

\section{Introducción}

Debido a que una de las principales dificultades a la hora de desarrollar la generación automática de textos se halla en la necesidad de preparar los datos de entrada, el presente trabajo modela el espacio de aplicación a partir de un conjunto de resultados simples de análisis morfológico en lenguaje natural, del que se ha eliminado toda redundancia para extraer su información esencial y obtener la estructura lógica de tal información - fase de análisis.

La producción textual estudia su obtención automática a partir de la representación interna de la inlormación - fase de generación. La principal aportación de este trabajo radica en pasar de esa estructura lógica a una frase variable — planificada en un único dominio limitado- con la que representar la información de forma cohesionada, coherente, comprensible y con cierto estilo. La posibilidad de decir lo mismo de diferentes formas obliga a seleccionar el léxico, a observar cl contexto y a reforzar el control mediante criterios de selección para asegurar que el texto obtenido sea correcto y resulte formal, objetivo y escueto. 
En la figura 1 se presentan las fases para la generación automática de las respuestas partiendo de las entradas —resultados simples de análisis morfológico.

Análisis

Generación

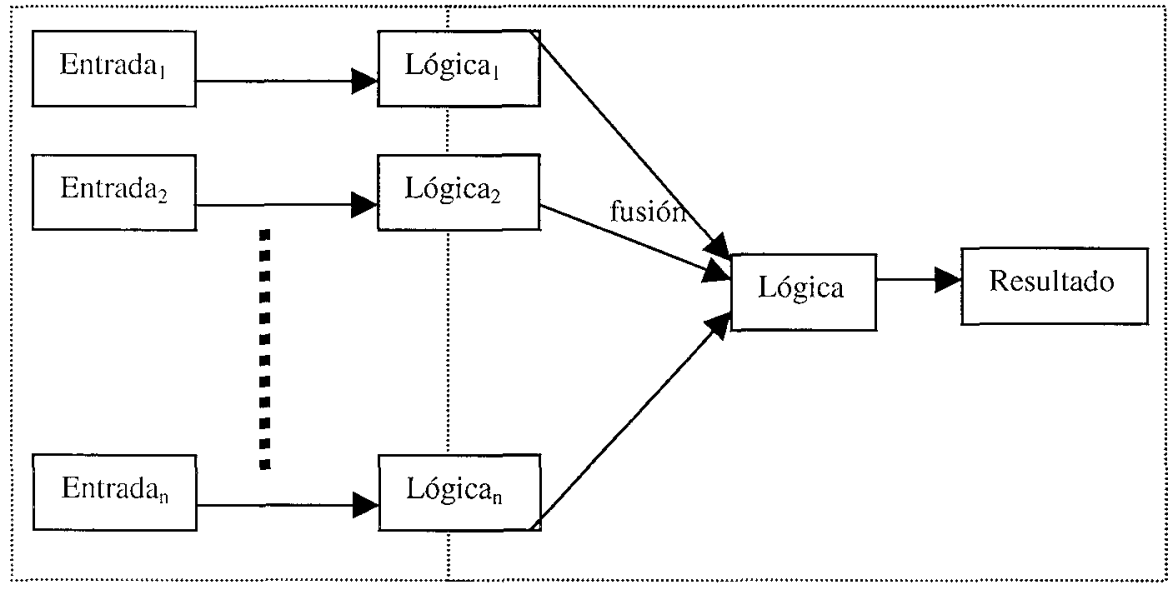

La ligura 2 muestra un sencillo paradigma de la generación de una respuesta al análisis morlólógico; las estructuras lógicas están formadas por una secuencia de palabras — se utiliza el símbolo ';' como separador de los elementos—, además se ha identificado un núcleo - - en cursiva y negrilla - y unos modificadores - sólo en cursiva - que dan cuentan de la informaciónsemántica a utilizar en el proceso de fusión.

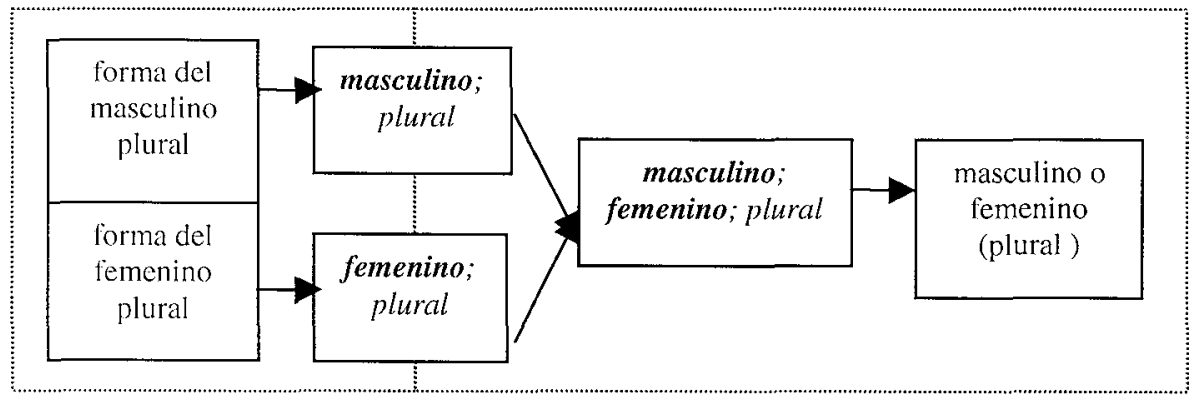

\section{Identificadores morfológicos simples}

Se parte de identificadores morfológicos simples que expresan las relaciones que interesan entre formas canónicas y formas flexionadas o derivadas en español. 
Las tablas 1-A y 1-B muestran los identificadores morfológicos simples del español considerados.

\begin{tabular}{|c|c|}
\hline \multicolumn{2}{|c|}{ Tabla 1-A: Identificadores simples de la conjugación } \\
\hline Formas simples & Formas compuestas \\
\hline 'infinitive' & 'infinitivo compuesto' \\
\hline 'germudio' & 'gerundio compuesto' \\
\hline "participio' & 'participio compuesto' \\
\hline '(P) persoma $(N)$ presente de indicativo' & '(P) persona ( $N$ ) preterito perfecto de indicativo' \\
\hline (P) persona ( $N)$ pretérito indefinido de indicativo" & '( $P$ ) persona $(N)$ pretérito anterior de indicativo' \\
\hline $\begin{array}{l}\text { "(P) persona (N) pretérito imperfecto de } \\
\text { indicativo' }\end{array}$ & $\begin{array}{l}\text { '(P) persona (N) pretérito pluscuamperfecto de } \\
\text { indicativo' }\end{array}$ \\
\hline (P) persona $(N)$ condicional simple' & '(P) persona $(N)$ condicional perfecto' \\
\hline '(P) persona (N) furturo imperfecto de indicativo' & '(P) persona ( $N)$ futuro perfecto de indicativo' \\
\hline '(P) persona (N) presente de subjuntivo' & "(P) persona $(N)$ preterito perfecto de subjuntivo' \\
\hline $\begin{array}{l}(P) \text { persona }(N) \text { pretérito imperfecto de } \\
\text { subjuntivo' }\end{array}$ & $\begin{array}{l}\text { 'P) persona ( } N) \text { pretérito pluscuamperfecto de } \\
\text { subjuntivo' }\end{array}$ \\
\hline "(P) persona (N) futuro imperfecto de subjuntivo' & '(P) persona $(N)$ futuro perfecto de subjuntivo' \\
\hline '( $\left(P^{\prime}\right)$ persona singular imperativo' & \\
\hline '(P) persona plaral imperativo' & \\
\hline & \\
\hline
\end{tabular}

\begin{tabular}{|c|}
\hline Tabla 1-B: Identificadores simples de la flexión y derivación \\
\hline formu canónica' \\
\hline forma del $(G)(N)$ \\
\hline forma del neutro $(N)$ \\
\hline forma del aumentativo $(G)(N)^{\prime}$ \\
\hline forma del diminutivo $(G)(N)$ \\
\hline 'forma del peyorativo $(G)(N)$ \\
\hline 'forma superiativa $(G)(N)$ de un adjetivo' \\
\hline 'aumentativo de adverbio' \\
\hline 'diminutivo de adverbio' \\
\hline 'peyorativo de adverbio' \\
\hline 'superlativo de adverbio' \\
\hline 'sustantivación femenino $(N){ }^{\prime}$ \\
\hline adjetivación invariante en género $(N)^{\prime}$ \\
\hline 'adjetivación superlativa $(G)(N)$ ' \\
\hline 'adverbialización de modo' \\
\hline 'adverbialización superlativa' \\
\hline Forma heteronimica $(G)^{\prime}$ \\
\hline 'diminutivo del gerundio' \\
\hline 'variante grafica' \\
\hline 'palabra significante en una frase' \\
\hline$(G)=$ masculino of femenino; $(N)=$ singular o plural \\
\hline
\end{tabular}




\section{Tratamiento de la conjugación}

Para generar el mensaje compuesto, a partir de un conjunto de identificadores simples de la conjugación, se seguirán los siguientes pasos:

\subsection{Fase de análisis:}

2.1.1. Creación de grupos. Cada identificador simple se desglosa en sus elementos primarios de información - estructura lógica. La tabla 2 muestra los grupos resultantes de procesar los identificadores simples de la conjugación.

\begin{tabular}{|c|c|}
\hline Identificaciones simples de la conjugación & Grupos resultantes \\
\hline 'infinitivo' & «infinitive» \\
\hline 'genundio' & "gerundio" \\
\hline participio' & "participio" \\
\hline '(P) persona $(N)$ presente de indicativo' & $\ll(P) ;$ persona; $(N) ;$ presente de indicativo» \\
\hline '(P) persona (N) pretérito indefinido de indicativo' & $\begin{array}{l}\text { «(P); persona; }(N) ; \text { pretérito indefinido de } \\
\text { indicativo» }\end{array}$ \\
\hline '(P) persona $(N)$ pretérito imperfecto de indicativo' & $\begin{array}{l}\text { "P); persona; }(N) ; \text { pretérito imperfecto de } \\
\text { indicativo» }\end{array}$ \\
\hline (P) persona $(N)$ condicional simple' & $«(P) ;$ persona; $(N) ;$ condicional simple» \\
\hline '(P) persona (N) futuro inperfecto de indicativo' & $\begin{array}{l}\text { "P } P \text {; persona; }(N) ; \text { futuro imperfecto de } \\
\text { indicativo» }\end{array}$ \\
\hline '(P) persona $(N)$ presente de subjuntivo' & «(P);persona; $(N) ;$ presente de subjuntivo" \\
\hline 'P) persona $(N)$ pretérito imperfecto de subjuntivo' & $\begin{array}{l}\text { «P); persona; }(N) ; \text { pretérito imperfecto de } \\
\text { subjuntivo» }\end{array}$ \\
\hline '(P) persona $(N)$ futuro imperfecto de subjuntivo' & $\begin{array}{l}\text { «(P); persona; }(N) ; \text { futuro imperfecto de } \\
\text { subjuntivo» }\end{array}$ \\
\hline '( $\left.P^{\prime}\right)$ persona singular imperativo' & "( $\left(P^{\prime}\right) ;$ persona; singular; imperativo» \\
\hline '(P) persona plural innerative' & «(P); persona; plural; imperativo» \\
\hline 'infinitivo compuesto' & «infinitivo compuesto» \\
\hline 'gerundio compuesto' & «gerundio compuesto» \\
\hline 'participio compuesto' & «participio compuesto» \\
\hline '(P) persoma $(N)$ preterito perfecto de indicativo' & $\begin{array}{l}\text { " }(P) \text {; persona; }(N) ; \text { preterito perfecto de } \\
\text { indicativo" }\end{array}$ \\
\hline '(P) personu (N) pretérito anterior de indicativo' & $\begin{array}{l}\text { " }(P) ; \text { persona; }(N) ; \text { pretérito anterior de } \\
\text { indicativo» }\end{array}$ \\
\hline $\begin{array}{l}\text { "}(P) \text { persona }(N) \text { pretérito pluscuamperfecto de } \\
\text { indicativo" }\end{array}$ & $\begin{array}{l}\text { "(P); persona; }(N) ; \text { pretérito pluscuatmperfecto } \\
\text { de indicativo» }\end{array}$ \\
\hline '(P) persona $(N)$ condicional perfecto' & $«(P) ;$ persona; $(N) ;$ condicional perfecto $»$ \\
\hline '(P) persona $(N)$ finturo perfecto de indicativo' & "(P);persona; $(N) ;$ fituro perfecto de indicativo» \\
\hline '(P) persoma $(N)$ preterito perfecto de subjuntivo' & $\begin{array}{l}\text { "P); persona; }(N) ; \text { preterito perfecto de } \\
\text { subjuntivo" }\end{array}$ \\
\hline $\begin{array}{l}\text { '(P) persona }(N) \text { preférito pluscuamperfecto de } \\
\text { subjuntivo' }\end{array}$ & $\begin{array}{l}\text { "P); persona; }(N) ; \text { pretérito pluscuamperfecto } \\
\text { de subjuntivo» }\end{array}$ \\
\hline '(P) persoma $(N)$ fituro perfecto de subjuntivo' & (P); persona; $(N) ;$ futuro perfecto \\
\hline
\end{tabular}




\begin{tabular}{|l|l|}
\hline Tabla 2: Grupos resultantes para los identificadores simples de la conjugación \\
\hline Identificaciones simples de la conjugación & Grupos resultantes \\
\hline & subjuntivo» \\
\hline$(P)=I^{\prime \prime}, 2^{\prime \prime} O 3^{\prime \prime} ;\left(P^{\prime}\right)=2^{\prime \prime} O 3^{\prime \prime} ;(N)=$ singular 0 plural \\
\hline
\end{tabular}

\subsection{Fase de generación:}

2.2. . Arreglos compactos de conjugación por extracción de factores comunes de los grupos. Se generan conforme a las siguientes consideraciones:

- Cuando un mismo tiempo aparece con distintos números y personas, se saca factor común el tiempo. A saber:

De « $I^{a}$; persona; singular; presente de indicativo» $+\left\langle 2^{a}\right.$; persona; plural; presente de indicativo»,

se obtienc « $I^{a}$; persona; singular; $y ; 2^{a}$; plural; presente de indicativo»; de coincidir cl número, también se extrae el número. A saber:

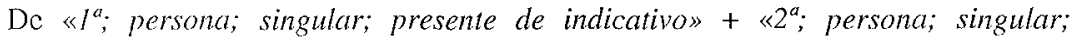
presente de indicativo»,

se obtienc « $1^{a}$ y $2^{a}$; persona; singular; presente de indicativo».

- Cuando un mismo tiempo y persona aparecen con distintos números, el tiempo y la persona devienen en factores comunes. A saber:

De « $I^{a}$; persona; singular; presente de indicativo» $+\left\langle 1^{a}\right.$; persona; plural; presente de indicativo»,

sc obtiene $~ « I^{a}$; persona; singular y plural; presente de indicativo».

- Cuando un mismo número y persona aparecen con distintos tiempos, el número y la persona representan el factor común. A saber:

Do $\ll 1^{a}$; persona; singular; presente de indicativo» $+\ll l^{a}$; persona; singular; condicional simple»,

se obtiene $« I^{a}$; persona; singular; presente de indicativo y condicional simple»;

de cambiar cl número, éste no forma parte del factor común. A saber:

De $\ll I^{a}$; persona; singular; presente de indicativo» $+\left\langle I^{a}\right.$; persona; plural; condicional simple»,

se obtiene " $l^{a}$; persona; singular; presente de indicativo; $y$; plural; condicional simple».

- Los grupos que no se hayan podido combinar se constituyen en arreglos compactos.

2.2.2. Generación del resultado morfológico de la conjugación. Por último, se combinan todos los arreglos compactos de conjugación utilizando el nexo ' $y$ ' y se climinan los delimitadores ';'.

\section{Tratamiento de la flexión y derivación}

A partir de un conjunto de identificadores simples de flexión y derivación, para generar el texto correspondiente a la flexión y derivación, se seguirán los siguientes pasos: 


\subsection{Fase de análisis:}

Creación de grupos. Cada identificador simple se desglosa en sus elementos primarios de información - estructura lógica- después de eliminar las palabras no relevantes; así, de 'Forma canónica' y 'Forma del masculino singular', se obtienen los grupos «canónica» y «masculino; singular». La tabla 3 muestra los grupos resultantes de la flexión y derivación.

\begin{tabular}{|c|c|}
\hline \multicolumn{2}{|c|}{$\begin{array}{l}\text { Tabla 3: Grupos resultantes para los identificadores simples de la flexión y } \\
\text { derivación }\end{array}$} \\
\hline $\begin{array}{l}\text { Identificaciones simples de la flexión y } \\
\text { derivación }\end{array}$ & Grupos resultantes \\
\hline 'forma canónicat' & «canónica» \\
\hline forma del $(G)(N)$ & $\ll(G) ;(N) »$ \\
\hline forma del neutro $(N)^{\prime}$ & «neutro; $(N)_{»}$ \\
\hline forma del aumentativo $(G)(N)$ & «aumentativo; $(G) ;(N) »$ \\
\hline 'forma del diminutivo $(G)(N)$ ' & «diminutivo; $(G) ;(N) »$ \\
\hline forma del peyorativo $(G)(N)$ & "peyorafivo; $(G) ;(N) »$ \\
\hline 'forma superlativa $(G)(N)$ de un adjetivo' & «superlativo; $(G) ;(N) ;$ un; adjetivo» \\
\hline 'clumentativo de adverbio' & «almentativo; adverbio» \\
\hline 'diminutivo de adverbio' & «diminutivo; adverbio» \\
\hline 'peyorativo de adverbio' & "peyorativo; adverbio» \\
\hline 'superlativo de adverbio' & «stuperlativo; adverbio» \\
\hline 'sustantivación femenino $(N)$ de un adjetivo' & «sustantivacionn; femenino; $(N) ;$ un; adjetivo» \\
\hline $\begin{array}{l}\text { "aljerivación invariante en género }(N) \text { de un } \\
\text { nombre' }\end{array}$ & "adjetivación; invariante; género; $(N)$; un; nombre» \\
\hline 'adjetivación fentenino $(N)$ ' & «adjetivación; femenino; $(N) »$ \\
\hline "adjetivación masculino plural" & "adjetivación; masculino; plural» \\
\hline 'adjetivación superlativa $(G)(N)$ ' & «adjetivación; superlativo; $(G) ;(N) »$ \\
\hline 'adverbializacion de modo' & "adverbializacion; modo» \\
\hline "adverbialización superlativa" & "adverbialización; superlativo» \\
\hline 'formal heteronímica $(G)^{\prime}$ & «heteronínica; $(G) »$ \\
\hline "diminutivo del gerundio' & «diminutivo; gerundio» \\
\hline 'variante grafica' & «variante gráfica \\
\hline 'palabra significante en tuna frase' & «palabra; significante; una; frase» \\
\hline
\end{tabular}

3.1.2. Identificación y catalogación de núcleos. Se denomina núcleo a los elementos de un grupo que recogen la información más relevante, y modificadores, al resto de sus elementos; los núcleos pueden ser simples - un solo elemento-o compuestos - varios separados por ' $;$ '. En la tabla 4 figura la clasificación en tres 
tipos de los núcleos como resultado del estudio de los identificadores simples de flexión y derivación.

\begin{tabular}{|l|l|}
\hline Tabla 4: Tipos de núcleos \\
\hline Tipo 0 & «anónica», «heteronímica», «variante gráfica», «significante» \\
\hline Tipo 1 & «masculino», «femenino», «neutro» \\
\hline Tipo 2 & $\begin{array}{l}\text { «adjetivación; superlativo; masculino», «adjetivación; superlativo; femenino», «adjetivación; } \\
\text { «aumentativo; masculino», «diminutivo; masculino», «peyorativo; masculino», «aumentativo; } \\
\text { femenino», «diminutivo; femenino», «peyorativo; femenino», «adjetivación», «sustantivación», } \\
\text { «aumentativo», «diminutivo», «peyorativo», «uperlativo», «adverbialización « }\end{array}$ \\
\hline
\end{tabular}

Para cada grupo, se determina su núcleo y se etiqueta según su tipo; de esta manera, el grupo «canónica» tiene como núcleo canónica y se etiqueta de tipo 0; para «masculino; singular» se toma como núcleo masculino y consta como de tipo 1 -palabras que indican género: masculino, femenino y neutro. Los núcleos compuestos más largos del tipo 2 tienen preferencia de elección en el análisis de núcleos: de «aumentativo; femenino», se considera como núcleo aumentativo; femenino - tipo 2 - y no aumentativo - tipo 2 - ni femenino tipo 1.

\subsection{Fase de generación:}

3.2.1. Unificación de grupos según núcleo. Se reúnen en un mismo arreglo compacto los grupos con el mismo núcleo o con la primera parte del mismo en común - caso de ser compuestos.

Primera vuclta: Se combinan los grupos cuyo núcleo coincida:

De «masculino; singular»+ «masculino; plural»,

sc obtiene «masculino; singular; Q; plural».

De «aumentativo; femenino; singular» + «aumentativo; femenino; plural», se obtiene «aumentativo; femenino; singular; $Q$; plural».

Nótese que se ha añadido el nexo ' $o$ ' para unir los modificadores, ya que el número no puede ser a la vez singular y plural.

Los grupos que no se hayan podido combinar se constituyen en arreglos compactos.

Segunda vuelta: Se combinan los arreglos compactos formados por núcleos de dos o tres elementos que tienen en común todos ellos menos el último; los elementos comunes pasan a ser el nuevo núcleo:

- Cuando coinciden los modificadores:

De «aumentativo; masculino; singular» + «aumentativo; femenino; singular», se obtiene «aumentativo; masculino; $Q$; femenino; singular».

Nótese que se ha añadido el nexo 'o' y el género será el nuevo modificador del núcleo y singular pasa a ser un modificador del género. 
- Cuando no coinciden los modificadores:

De «aumentativo; masculino; singular» + «aumentativo; femenino; plural», se obtiene «aumentativo; masculino; singular;, ; femenino; plural».

Nótese que el nexo ' 0 ' se incluye para unir los nuevos grupos de modificadores «masculino; singular»y «femenino; plural».

3.2.2. Fusión según tipo de núcleo. Se reúnen los arreglos compactos con núcleos del mismo tipo.

- Cuando coinciden los modificadores:

De «masculino; singular $+\langle$ femenino; singular»,

se obtiene «masculino; $\underline{Q}$; fernenino; singular».

- Cuando no coinciden los modificadores:

Dc «masculino; singular» + «femenino; plural»,

se obtiene «masculino; singular; 2 ; femenino; plural» - el grupo pasa a tener dos núcleos con sus respectivos modificadores unidos por 'o'.

3.2.3. Inclusión de paréntesis. Se encierran entre paréntesis los modificadores de cada núcleo:

- Cuando el núcleo es simple y tiene asociado más de un modificador:

De «masculino; singular; o; plural»,

se obtiene «masculino; (singular; o; plural 2 ».

Téngase en cuenta que en un mismo arreglo compacto puede haber varios núcleos simples.

De «femenino; singular; o; plural; o; masculino; singular»,

Se obtiene «femenino; (singular; o; plural); o; masculino; singular».

- Cuando el núcleo es compuesto, con independencia del número de modificadores:

De «masculino; o; femenino; singular», se obtiene «masculino; o; femenino; (singular)».

- Cuando existen modificadores de los modificadores:

De «aumentativo; masculino; o; femenino; singular»,

se obtiene «aumentativo; (masculino; o; femenino; (singular) $) 》$.

3.2.4. Generación del resultado morfológico de la flexión y de la derivación. Se obtienc al combinar todos los arreglos compactos de flexión y derivación en orden ascendente del tipo del núcleo y al eliminar los delimitadores ';'.

- Cuando un arreglo es «canónica» o contiene «heteronímica» se antepone 'forma'.

- Cuando hay más de un arreglo con núcleos del tipo 0 se unen mediante el nexo de unión ' $y$ '.

«variante gráfica» + «palabra; significante; en una frase» $\Rightarrow$

$\Rightarrow$ «variante gráfica $\$ palabra significante en una frase»

- Cuando se une un arreglo con núcleo «canónica» con un arreglo con núcleo del tipo 1, éste debe ir entre paréntesis.

«anónica» $+\langle$ masculino; singular» $\Rightarrow\langle$ forma canónica _masculino singular)» 
- Cuando cxiste anidamiento de paréntesis, se resuelve eliminando el más interno e incluyendo una ',' para mantener la delimitación.

«Canónica»+ «masculino; (singular; o; plural)» $\Rightarrow$

$\Rightarrow \ll$ forma canónica (masculino (singular o plural) $\gg \Rightarrow$

$\Rightarrow$ «orma canónica _masculino, singular o plural $\rangle_{\star}$

«aumentativo; (masculino; o; femenino; (singular))» $\Rightarrow$

$\Rightarrow$ «alumentativo (masculino o femenino ${ }_{2}$ singular)»

- Ei resto de arreglos con núcleos de distinto tipo y los de tipo 0 entre sí se unen mediante el nexo de unión ' 0 '.

«canónica» + «aumentativo; femenino; plural» $\Rightarrow$

$\Rightarrow$ «forma canónica @ aumentativo femenino plural»

- Ante 'adverbio', 'modo' , 'un' o 'una' se pone 'de'.

«superlativo; adverbio» $\Rightarrow$ «superlativo de adverbio»

«superlativo; masculino, singular; un; adjetivo» $\Rightarrow$ «superlativo masculino singular de un adjetivos

\section{Respuesta final}

En general, la respuesta morfológica final se corresponde con el resultado morfológico de la conjugación o con el de la flexión y derivación; en caso de existir ambos, se unen mediante el nexo ' 0 ' para obtener la respuesta final.

\section{Ejemplos}

5.1. Ejemplo hipotético para poner de manifiesto cómo actúa el algoritmo desarrollado con los identificadores simples de conjugación para obtener un arreglo completo de conjugación:

Identilicadores simples de conjugación de partida:

l. ' ' ${ }^{a}$ persona singular presente de indicativo'

2. '2" persona singular presente de indicativo'

3. ' ' 3 a persona plural presente de indicativo'

4. '2a persona singular futuro imperfecto de indicativo'

5. '2" persona singular imperativo'

5.1.1. Creación de grupos resultantes de conjugación:

l. « $l^{a}$; persona; singular; presente de indicativo»

2. "2a; persona; singular; presente de indicativo»

3. « $3^{a}$; persona; plural; presente de indicativo»

4. «2a; persona; singular; futuro imperfecto de indicativo»

5. «2a; persona; singular; imperativo» 
5.1.2. Arreglos compactos de conjugación:

De « ${ }^{\mathrm{a}}$; persona; singular; presente de indicativo» $+\ll 2^{\mathrm{a}}$; persona; singular; presente de indicativo»,

se obtiene $\left\langle 1^{\mathrm{a}} \bigvee 2^{\mathrm{a}}\right.$; persona; singular; presente de indicativo».

De $\ll 1^{a}$ y $2^{a}$; persona; singular; presente de indicativo» $+\ll 3^{\text {a }}$; persona; plural; presente de indicativo»,

se obtiene « $1^{\mathrm{a}}$ y $2^{\mathrm{a}}$; persona; singular; $y ; 3^{\mathrm{a}}$; plural; presente de indicativo».

De $\ll 2^{\mathrm{a}}$; persona; singular; futuro imperfecto de indicativo» $+\left\langle 2^{\mathrm{a}}\right.$; persona; singular imperativo»,

sc obtiene $\ll 2^{a}$; persona singular; futuro imperfecto de indicativo e imperativo».

5. 1.3. Generación del arreglo completo de conjugación:

De « $1^{\text {" }}$ y $2^{\mathrm{a}}$; persona; singular; $y ; 3^{\mathrm{a}}$; plural; presente de indicativo»

$y$ de $« 2^{a}$; persona singular; futuro imperfecto de indicativo e imperativo»,

se obtiene « $1^{\mathrm{a}}$ y $2^{\mathrm{a}}$ persona singular y $3^{\mathrm{a}}$ plural presente de indicativo $y 2^{\mathrm{a}}$ persona singular futuro imperfecto de indicativo e imperativo».

5.2. Ejemplo hipotético para poner de manifiesto cómo actúa el algoritmo desarrollado con los identificadores simples de flexión y derivación para obtener un arreglo completo de flexión y derivación:

Identilicadores simples de flexión y derivación de partida:

1. 'forna canónica'

2. 'forma del masculino plural'

3. 'forma del masculino singular'

4. 'forma del femenino plural'

5. 'forma del femenino singular'

6. 'forma del diminutivo femenino singular'

7. 'forma del diminutivo masculino singular'

8. 'forma del aumentativo femenino singular'

9. 'forma del aumentativo masculino plural'

5.2.1. Creación de grupos resultantes de conjugación:

1. «canónica»

2. «masculino; singular»

3. «masculino; plural»

4. «femenino; singular»

5. «femenino; plural»

6. «diminutivo; femenino; singular»

7. «diminutivo; masculino; singular»

8. «aumentativo; femenino; singular»

9. «aumentativo; masculino; plural» 
5.2.2. Identificación y catalogación de los núcleos:

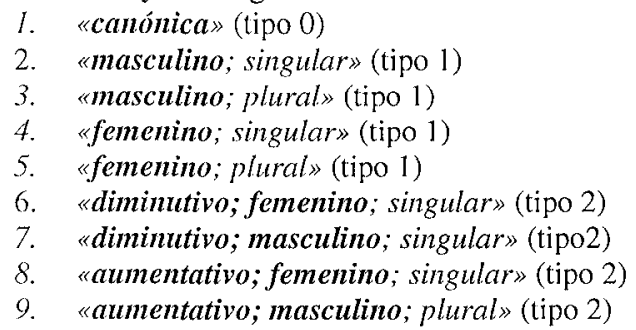

5.2.3. Unificación de grupos según núcleo:

Primera vuelta (núcleos coincidentes):

De «masculino; singular» + «masculino; plural»

se obtiene «masculino; singular; Q; plural»

y de «femenino; singular» + «emenino; plural»

se obtiene «femenino; singular; $\underline{Q}$; plural»

Segunda vuelta (núcleos parcialmente coincidentes):

De «diminutivo; femenino; singular» + «diminutivo; masculino; singular»

sc obtienc «diminutivo; femenino; Q; masculino; singular ;

y de «aumentativo; lemenino; singular» + «aumentativo; masculino; plural»

se obtiene «aumentativo; femenino; singular; $\underline{Q}$; masculino; plural»

Así pues, ahora quedan los siguientes arreglos compactos:

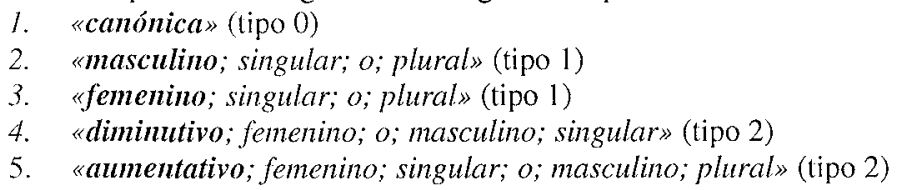

5.2.4. Fusión según tipo de núcleo:

De «masculino; singular; o; plural + «femenino; singular; o; plural»,

se obticne «masculino; $\boldsymbol{\alpha}$; femenino; singular; o; plural».

Nótese que como los modificadores coinciden en ambos arreglos será necesario unir sus núcleos con $\mathrm{cl}$ nexo ' $\mathrm{O}$ '.

De «diminutivo; femenino; o; masculino; singular»

y de «aumentativo; femenino; singular o; masculino; plural»,

se obticne «diminutivo; femenino; o; masculino; singular; $\Omega$; aumentativo; fenenino; singular o; masculino; plural»

Nótese que como los modificadores no coinciden, se unen los dos arreglos con una ' $O$ '.

Quedan los arreglos compactos:

l. «canónica» (tipo 0) 
2. «masculino; o; femenino; singular; o; plural» (tipo 1)

3. «diminutivo; femenino; o; masculino; singular; $o$; aumentativo; femenino; singular o; masculino; plural» (tipo 2)

5.2.5. Inclusión de paréntesis.

1. «canónica» (tipo 0)

2. «masculino; o; femenino; (singular; o; plural)» (tipo 1)

3. «diminutivo; (femenino; o; masculino; (singular)); o; aumentativo; (femenino; singular o; masculino; plural)» (tipo 2)

5.2.6. Generación del arreglo completo de flexión y derivación.

De «canónica» + «masculino; o; femenino; (singular; o; plural)», sc obtiene «forma canónica (masculino o femenino singular o plural)»; y al añadir «diminutivo; (femenino; 0 ; masculino; (singular)); 0 ; aumentativo; (femenino; singular o; masculino; plural)»,

se obtiene «forma canónica (masculino o femenino, singular o plural) a diminutivo (femenino o masculino, singular) o aumentativo (femenino singular o masculino plural)».

5.3. En el caso de darse simultáneamente el resultado morlológico de la conjugación y el de la flexión y derivación, se unen ambos resultados mediante el nexo ' $O$ ' para obtener la respuesta final. Con los ejemplos vistos será:

De « $1^{a}$ y $2^{a}$ persona singular y $3^{a}$ plural presente de indicativo y $2^{a}$ persona singular futuro imperfecto de indicativo e imperativo»

$\mathrm{y}$ de «forma canónica (masculino o femenino, singular o plural) o diminutivo (femenino o masculino, singular) o aumentativo (femenino singular o masculino plural)»,

se obtiene « $l^{a}$ y $2^{a}$ persona singular y $3^{a}$ plural presente de indicativo y $2^{a}$ persona singular futuro imperfecto de indicativo e imperativo o forma canónica (masculino o femenino, singular o plural) o diminutivo (femenino o masculino, singular) o aumentativo (femenino singular o masculino plural)»

\section{Conclusiones}

En el presente artículo se resuelve el problema de la generación monofrase en un entorno bien definido, capaz de obtener mensajes flexibles sin necesidad de almacenar todas las posibilidades; se ha logrado una metodología para la producción automática de la respuesta al análisis morfológico de cualquier palabra del español a partir de los identificadores morfológicos simples, utilizando una información semántica mínima. Aunque muchas de las combinaciones contempladas no ocurren en la gramática española, los pasos seguidos fueron: estudio de las posibles entradas, estilo del texto de salida, agrupación de las 
entradas en niveles semánticos — tipos de núcleos- y procesos de análisis y generación, lo que puede dar una visión inicial del camino a seguir para futuros trabajos en la generación automática de textos.

En próximos trabajos se podrá adaptar esta metodología a otros dominios para cubrir la amplitud de sus respuestas — producción de mensajes que impliquen categorías gramaticales. Otra línea de investigación podría evolucionar hacia la generación para casos multifrase, donde sería necesario tener en cuenta la relación entre las diferentes frases del discurso, lo que añade un componente semántico mayor.

\section{Referencias bibliográficas:}

Gómez Guinovart, J. (1999): Las escritura asistida por ordenador. Problemas de sintaxis y de estilo, Vigo: Servicio de Publicaciones de la Universidad de Vigo.

Pérez Aguiar, J. (1996): Reconocimiento y generación integrada de la morfología del español: Una aplicación a la gestión de un diccionario de sinónimos y antónimos, Tesis para la obtención del Título de Doctor en Informática. Universidad de Las Palmas de Gran Canaria.

Santana, O.; Pérez, J.; Carreras, F.; Duque, J.; Hernández, Z.; Rodríguez, G. (1999): «FLANOM: Flexionador y lematizador automático de formas nominales». Lingiiística Española Actual, XXI, 2, págs. 1-46.

Santana, O.; Pérez, J.; Hernández, Z.; Carreras, F.; Rodríguez, G. (1997): «FLAVER: Flexionador y lematizador automático de formas verbales», Lingïistica Española Actual, XIX, 2, págs. 229-282.

Zaenen, A.; Uszkoreit, H.; Karlsson, F.; Karttunen, L.; Sanfilippo, A.; Pulman, S. G.; Pereira, F.; Briscoe, T. (1995): «Survey of the State of the Art in Human Language Technology: Language Analysis and Understanding». National Science Foundation. Directorate XIII-E of the Commission of the European Communities. Center for Spoken Language Understanding, Oregon Graduate Institute. Noviembre. 